\title{
Coherence Evaluation of TanDEM-X Interferometric Data
}

\author{
Michele Martone, Benjamin Bräutigam, Paola Rizzoli, Carolina Gonzalez, \\ Markus Bachmann, Gerhard Krieger \\ Microwaves and Radar Institute, German Aerospace Center (DLR), \\ Oberpfaffenhofen, Germany. \\ Email: Michele.Martone@dlr.de
}

\begin{abstract}
The TanDEM-X (TerraSAR-X add-on for Digital Elevation Measurement) mission comprises two nearly identical satellites: TerraSAR-X (TSX, launched in 2007), and TanDEM-X (TDX, launched in June 2010). The primary objective of the mission is to generate a worldwide and consistent digital elevation model (DEM) with an unprecedented accuracy. During the first three months after its launch, the TDX satellite was tested and calibrated in monostatic configuration with both satellites flying in $20 \mathrm{~km}$ along-track distance, and it was proven that the system and acquisition performance is almost identical to TSX. Both satellites were then brought into close formation of a few hundred meters distance to begin the bistatic commissioning phase. Since then, TSX and TDX have acted as a large single-pass radar interferometer, which overcomes the limitations imposed by repeat-pass interferometry and allow the acquisition of highly accurate cross- and along-track interferograms. In December 2010, TanDEM-X began with operational global acquisition: bistatic and monostatic SAR images are simultaneously acquired in stripmap mode and processed to interferograms, from which a global DEM is derived. The key parameter in estimating interferometric performance is the coherence, which is deeply evaluated in this paper. The impact of different decorrelation sources as well as the performance stability over time is investigated by means of statistical analyses and dedicated acquisitions on defined test sites, demonstrating the outstanding interferometric capabilities of the TanDEM-X mission.
\end{abstract}

Keywords: Synthetic aperture radar (SAR), bistatic SAR, interferometry, coherence, digital elevation model (DEM), TanDEM-X. 


\section{Introduction}

In the last decades, an increasing interest has arisen to use Synthetic Aperture Radar (SAR) systems in remote sensing applications. Radar sensors can monitor their target continuously and without cloud disturbance, unlike optical sensors, which need the sunlight to be able to record the area of interest. In this context, Interferometric SAR (InSAR) exploits the phase difference of at least two complex SAR images, acquired from different orbit positions and/or at different times. This derived information allows the estimation and assessment of many geophysical parameters, such as ocean currents, ground deformations, and Earth's topography by generation of digital elevation models (DEMs). InSAR represents nowadays a well-recognized and powerful instrument and DEMs are widely employed in many commercial and scientific applications, such as, for example, Geographic Information System (GIS), Global Positioning System (GPS), as well as many geoscience fields, like geology, physical geography, glaciology, and oceanography (Moreira et al., 2004; Zebker et al., 1994). In 2000, the Shuttle Radar Topography Mission (SRTM) created the first near-global data set of land elevations (Werner, 2001). The scientific community as well as commercial customers have then clearly shown the demand for more accurate interferometric products covering the entire land mass. TanDEM-X (TerraSAR-X add-on for Digital Elevation Measurement) is the first operational spaceborne bistatic SAR system comprising two twin satellites: TerraSAR-X (TSX, launched in 2007), and TanDEM-X (TDX, launched in June 2010). During the first three months after its launch, the TDX satellite was tested and calibrated in monostatic configuration with the two satellites flying in $20 \mathrm{~km}$ alongtrack distance. It was proven that the system and acquisition performance are very similar to TSX. In October 2010, TSX and TDX were brought into close formation of a few hundreds meter distance. Since then, TSX and TDX act as a large single-pass radar interferometer, with the opportunity for flexible along- and across-track baseline selection. In the bistatic Commissioning Phase (CP) first interferometric capabilities and performance were assessed and acquisitions were operationally performed in bistatic configuration. Here, either TSX or TDX is used for transmission and both satellites record simultaneously the signal backscattered from the Earth's surface. In December 2010, TanDEM-X began with operational global acquisition. Bistatic and 
monostatic SAR images are simultaneously acquired in stripmap mode and processed to single-pass across-track interferograms. In this context, a major challenge is the synchronization of the ultra-stable oscillators (USO) operating independently on the two satellites. Smallest differential deviations in terms of phase and frequency lead to considerable phase errors and geometrical image distortions. Therefore, the two satellites directly exchange so called synchronization pulses, and, based on these, the differential phase noise and frequency deviation of the oscillators are compensated on-ground (Breit et al., 2011). Another fundamental aspect in bistatic SAR data processing is the baseline calibration concept. Few millimeters baseline estimation uncertainty (mainly in cross-track and radial direction) involves vertical and horizontal DEM displacement in the order of meters. For the TanDEM-X mission, baseline calibration is performed by a double differential evaluation of the GPS carrier phase measurements (Hueso et al., 2011).

The primary objective of the mission is the generation of a global, consistent and high-precision DEM (Krieger et al., 2007). This goal will be achieved by performing at least two global mappings of the Earth's landmass. The two acquisition sets use mutually displaced beams and different baselines, to further improve the performance and to keep it almost constant over range. In Table 1 the specifications for SRTM (Rodriguez et al., 2006) and TanDEM-X (DEM products specification, 2012) missions are compared.

One of the key parameters used to evaluate quality and performance of interferometric products is the coherence $\gamma$. It represents the normalized complex correlation coefficient between master and slave acquisition and gives information about the amount of noise in the interferogram (Bamler and Hartl, 1998; Zebker and Villasenor, 1992). Several error sources may contribute to coherence loss

$$
\gamma=\gamma_{S N R} \cdot \gamma_{\text {Vol }} \cdot \gamma_{\text {Quant }} \cdot \gamma_{A m b} \cdot \gamma_{\text {Range }} \cdot \gamma_{\text {Azimuth }} \cdot \gamma_{\text {Temp }},
$$

where the terms on the right-hand side describe decorrelation due to: limited signal-to-noise ratio $\left(\gamma_{S N R}\right)$, volume decorrelation $\left(\gamma_{V o l}\right)$, quantization errors $\left(\gamma_{\text {Quant }}\right)$, ambiguities $\left(\gamma_{A m b}\right)$, baseline decorrelation $\left(\gamma_{\text {Range }}\right)$, errors due to relative shift of Doppler spectra $\left(\gamma_{\text {Azimuth }}\right)$, temporal decorrelation $\left(\gamma_{\text {Temp }}\right)$. As TanDEM-X operates as a single-pass radar interferometer, coherence loss caused by a change of the scatterer structure is negligible (i.e. $\gamma_{T e m p}=1$ ), which is a valid assumption for most types of land coverage. By looking at first statistics on phase unwrapping indicators, coherence of 0.6 provides 
an already acceptable interferometric quality. In more than $90 \%$ of these cases, phase residues of smaller than $1 \%$ have been observed, which provides a robust unwrapping quality according to (Suchandt and Eineder, 2003).

Another important parameter is represented by the height of ambiguity (HoA), which is the height difference corresponding to a complete $2 \pi$ cycle of the interferometric phase, and tells about the phase-to-height sensitivity in the interferogram. For the bistatic case, it is defined as follows

$$
\mathrm{HoA}=\frac{\lambda r \sin \left(\theta_{i}\right)}{\mathrm{B}_{\perp}}
$$

being $\lambda$ the radar wavelength, $r$ the slant range, $\theta_{i}$ the incidence angle, and $B_{\perp}$ the baseline perpendicular to the line of sight.

The knowledge of the coherence allows for the derivation of the interferometric phase errors $\Delta \varphi$, that directly depend also on the equivalent number of looks (Lee et al., 1994). Defining $\Delta \varphi_{90 \%}$ as the $90 \%$ point-to-point phase error, the relative height error as required by the TanDEM-X DEM specifications may be finally expressed as

$$
\Delta h_{90 \%}=\operatorname{HoA} \cdot\left(\Delta \varphi_{90 \%} / 2 \pi\right) .
$$

For the first global acquisition of TanDEM-X, the height of ambiguity was typically between $45 \mathrm{~m}$ and $60 \mathrm{~m}$, ensuring good unwrapping quality over most land types. For the second global DEM, larger baselines will be considered (HoA of about $35 \mathrm{~m}$ ). The combination of at least two acquisitions by means of multi-baseline phase unwrapping algorithms (Lachaise et al., 2007), will then allow to fully meet the mission requirements.

This paper focuses on the coherence evaluation from interferometric TanDEM-X data acquired during the bistatic Commissioning Phase and the first year of the operational phase, and is organized as follows. First, the impact of limited SNR is investigated on different land covers. Volume decorrelation effects are evaluated in Section 3, and consequent implications on the global acquisition plan are described. In Section 4 quantization effects on coherence are assessed. Coherence losses due to azimuth ambiguities are investigated in Section 5. A detailed analysis of coherence dependence on polarization is provided in Section 6. Section 7 presents the results of the long-term repeatpass acquisitions on defined test sites, showing the influence of the acquisition geometry on interferometric performance, and proving a substantial stability over time of TanDEM-X. In Section 8 the current status of global coherence 
quality is given. This paper is concluded in Section 9 with a short summary and outlook.

Table 1: Comparison of SRTM and TanDEM-X specifications.

\begin{tabular}{|c|c|c|c|}
\hline Requirement & Specification & SRTM & TanDEM-X \\
\hline Relative Vertical Accuracy & $\begin{array}{c}90 \% \text { linear point-to-point } \\
\text { error over a } 1^{\circ} \mathrm{x} 1^{\circ} \text { cell }\end{array}$ & $\begin{array}{c}12 \mathrm{~m} \text { (slope }<20 \%) \\
15 \mathrm{~m}(\text { slope }>20 \%)\end{array}$ & $\begin{array}{c}2 \mathrm{~m} \text { (slope }<20 \%) \\
4 \mathrm{~m}(\text { slope }>20 \%)\end{array}$ \\
\hline Absolute Vertical Accuracy & $90 \%$ linear error & $18 \mathrm{~m}$ & $10 \mathrm{~m}$ \\
\hline Absolute Horizontal Accuracy & $90 \%$ circular error & $23 \mathrm{~m}$ & $10 \mathrm{~m}$ \\
\hline Spatial Resolution & independent pixels & $30 \mathrm{~m}$ & $12 \mathrm{~m}$ \\
& & & $(0.4$ arc sec at equator at equator $)$ \\
\hline
\end{tabular}

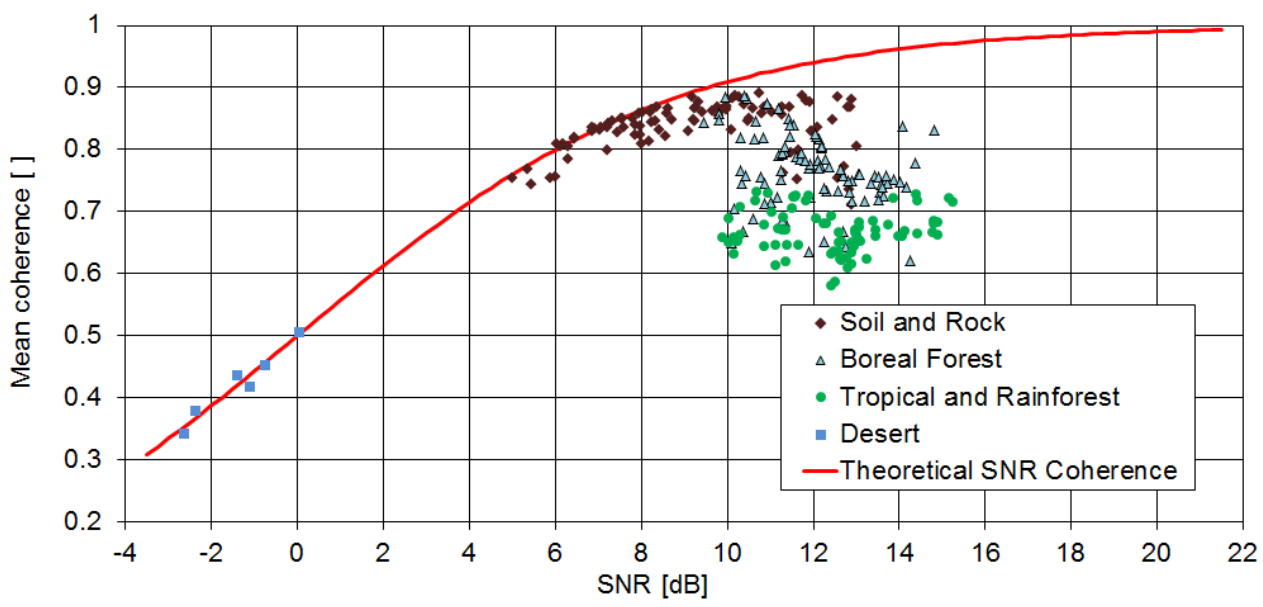

Figure 1: Interferometric coherence over Signal-to-Noise Ratio (SNR). The red line indicates the theoretical SNR coherence, as defined in (5).

\section{SNR Decorrelation}

The signal-to-noise ratio (SNR) is a measure to describe the image quality of remote sensing systems, estimating how much a signal has been corrupted by noise. From a SAR image, the SNR is computed as follows:

$$
\mathrm{SNR}=\frac{\beta_{0} \cdot \sin \left(\theta_{i}\right)-\mathrm{NESZ}}{\mathrm{NESZ}}
$$


where $\beta_{0}$ is the calibrated radar brightness, $\theta_{i}$ is the incidence angle, and NESZ stands for Noise Equivalent Sigma Zero, and describes the influence of noise contributions, like antenna pattern, instrument thermal noise, and processing filters. On TSX and TDX, the NESZ is estimated by means of receive-only pulses, which are commanded during the data-take.

In order to investigate the effects of SNR on interferometric performance, single-pass interferograms acquired over test sites showing different vegetation and soil characteristics have been analyzed. In the following, the term acquisition will refer to the entire data take, whereas a processed scene extends usually for an azimuth length of about $50 \mathrm{~km}$. Therefore, for longer acquisitions more scenes can be obtained. As the SNR difference between the two channels is very small (always less than $1 \mathrm{~dB}$ ), only the master channel has been considered. In Figure 1, the interferometric coherence over SNR is depicted: for most of the land cover types an SNR always higher than 6 $\mathrm{dB}$ is noticed, and quite stable interferometric performance is obtained, with a coherence almost always greater than 0.6 , providing in most cases sufficient phase unwrapping quality. The results are divided into four main land classes: boreal forest (light blue triangles) and soil and rock (brown rhombi) test sites have on average better performance $(\gamma>0.7)$ than areas characterized by tropical or rainforest (marked in green). Here, coherence losses are mainly due to the existence of a scattering volume over dense forest (the so-called volume decorrelation, deeply investigated in the next section). The red line on the top of the figure indicates the theoretical SNR coherence, i.e. the expected coherence as if the finite sensitivity of the receiving antenna was the only error source (Just and Bamler, 1994; Zebker and Villasenor, 1992), defined as

$$
\gamma_{\mathrm{SNR}}=\frac{1}{\sqrt{1+\mathrm{SNR}_{\mathrm{TSX}}^{-1}} \cdot \sqrt{1+\mathrm{SNR}_{\mathrm{TDX}}^{-1}}} \stackrel{\mathrm{SNR}_{\mathrm{TSX}} \cong \mathrm{SNR}_{\mathrm{TDX}}=\mathrm{SNR}}{=} \frac{1}{1+\mathrm{SNR}^{-1}},
$$

and proves, indeed, the presence of additional decorrelation phenomena as expected from (1). On the other hand, performance over sandy desert (blue squares) is strongly affected by the weak power of the backscattered signal from sand (SNR decorrelation): for SNR smaller than $2 \mathrm{~dB}$ a coherence rarely above 0.5 can be observed. In Figure 2, as an example, the coherence maps for two bistatic scenes over the Namibia desert are shown. For the image on the left side, the incidence angle is of about $48^{\circ}$, for the one on the right side it is $32^{\circ}$. The $\mathrm{SNR}$ is about $3 \mathrm{~dB}$ higher in the right image, and a 

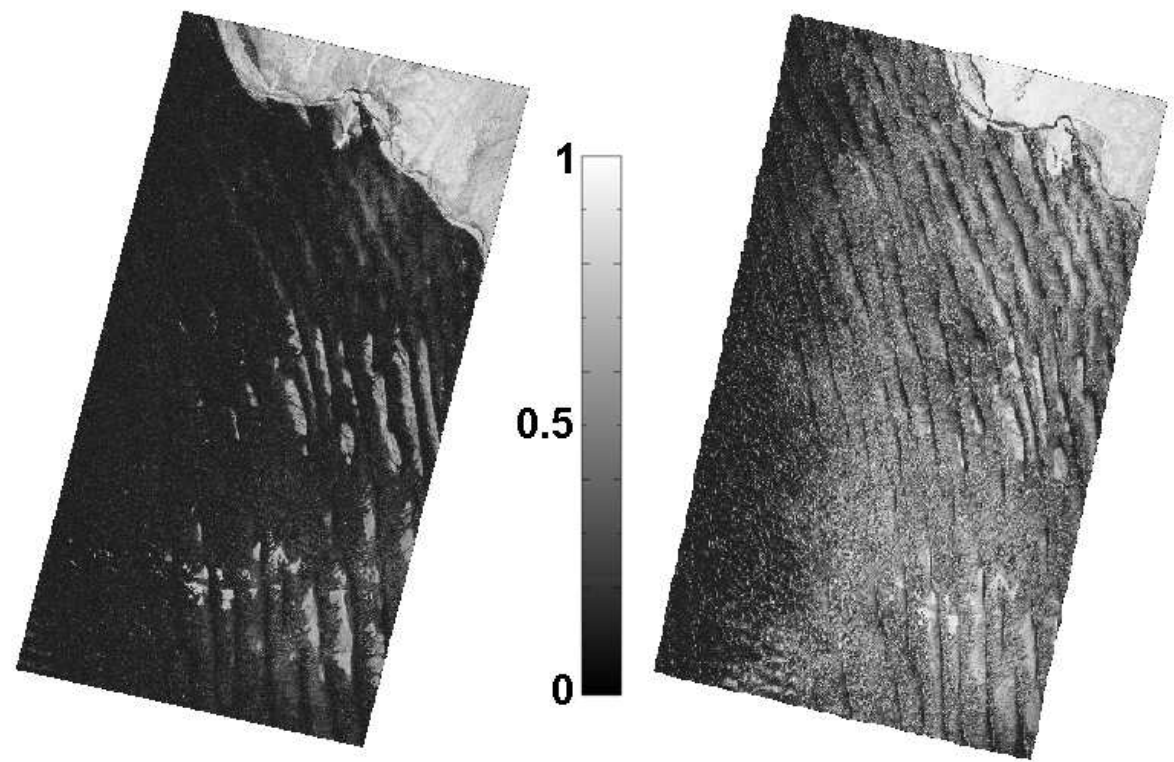

Figure 2: Coherence maps over Namibia desert; (left) HH polarization, HoA: $45 \mathrm{~m}\left(\mathrm{~B}_{\perp}\right.$ : $186 \mathrm{~m}$ ), $\theta_{i}: 47.7^{\circ}$, mean coherence over sand $\sim 0.25$; (right) HH polarization, HoA: $26 \mathrm{~m}$ $\left(\mathrm{B}_{\perp}: 162 \mathrm{~m}\right), \theta_{i}: 32.4^{\circ}$, mean coherence over sand $\sim 0.41$. On the rocky area, the mean coherence is $\sim 0.7$ for both images.

coherence improvement of about $60 \%$ (from 0.25 to 0.41 ) is obtained over the areas covered by sand, where over the rocky region (in the upper part of the images) almost the same performance is observed. Therefore, optimization of the imaging geometry, by employment of even steeper incidence angles, will be further investigated to minimize the performance loss over low backscatter areas. Moreover, the influence of other aspects which might affect the NESZ estimation as, e.g., data quantization, is currently being examined.

\section{Volume Decorrelation}

On vegetated areas, volume scattering is an important source of coherence loss (Treuhaft and Siqueira, 2000). The effect of volume scattering is similar to the classical baseline decorrelation: the existence of multiple scatterers at different heights and within a single resolution cell results in an increase of the interferometric phase uncertainty. In order to evaluate volume decorrelation, both statistical analyses and dedicated acquisitions over defined test sites with different vegetation characteristics were carried out. As an example, 

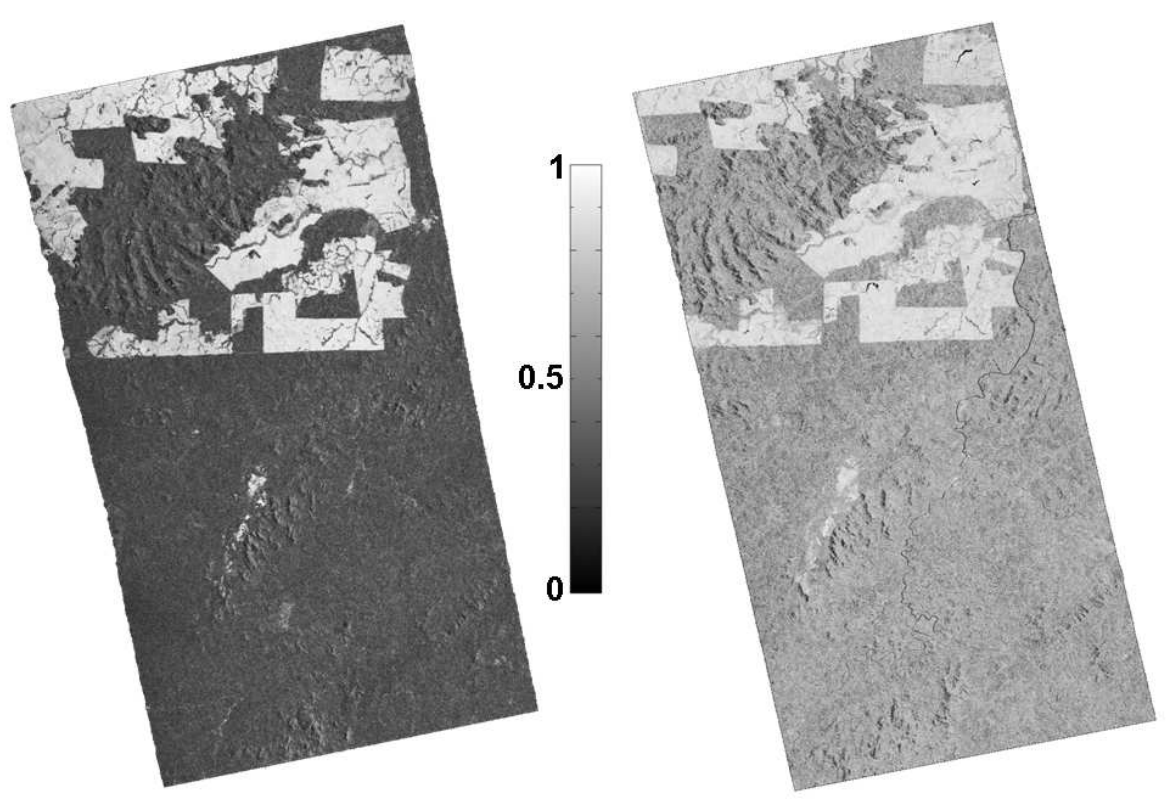

Figure 3: Coherence maps over Amazon rainforest; (left) HH polarization, HoA: $25 \mathrm{~m}$ $\left(B_{\perp}: 182 \mathrm{~m}\right), \theta_{i}: 29.9^{\circ}$, mean coherence over forest $\sim 0.3$; (right) HH polarization, HoA: $52 \mathrm{~m}\left(\mathrm{~B}_{\perp}: 162 \mathrm{~m}\right), \theta_{i}: 47.7^{\circ}$, mean coherence over forest $\sim 0.6$. On clear-cuts, the mean coherence is $\sim 0.8$ for both images.

Figure 3 depicts the coherence maps for two interferometric scenes acquired over the same area in the Amazon rainforest. For the image on the left side, the height of ambiguity is $25 \mathrm{~m}$, for the one on the right side it is $52 \mathrm{~m}$. The improvement is particularly visible over the forested areas (lower part of the image), whereas for clear-cuts almost the same performance is obtained. Results from 3074 bistatic scenes acquired over the Amazon rainforest in the period between October 2010 and June 2011 are shown in Figure 4. In the lower right corner, the considered area is highlighted, which includes a variety of sites with different forest density as well as topographic conditions. For each scene, the mean coherence over land has been evaluated, by applying a water mask. Test acquisitions with HoA smaller than 38-39 m (left part of the image) were commanded during the bistatic Commissioning Phase, and a limited interferometric performance can be observed, with a coherence rarely above 0.6. For this reason, a new target height of ambiguity for the first global DEM acquisition was set to $45 \mathrm{~m}$ (initially set at $40 \mathrm{~m}$ ). The corresponding points in Figure 4 lie between the two red lines. A mean co- 


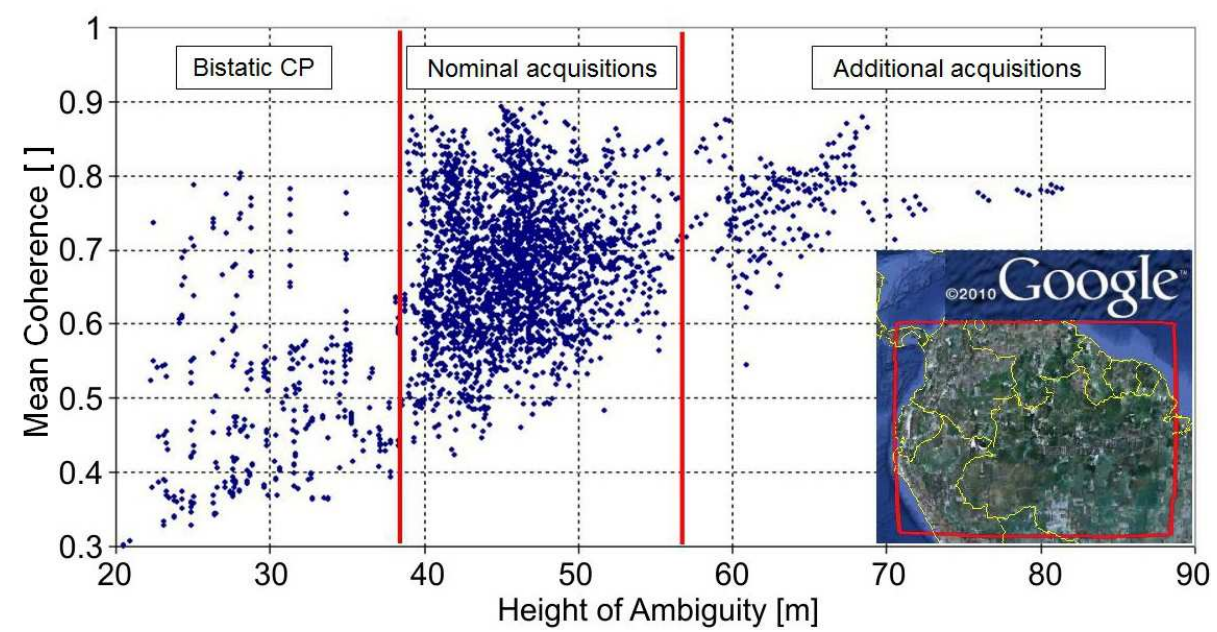

Figure 4: Mean coherence over height of ambiguity (HoA) for 3074 bistatic scenes acquired over Amazon rainforest (the considered area is highlighted in the lower right corner). Test acquisitions with HoA $<38 \mathrm{~m}$ (during the bistatic Commissioning Phase, left part of the image) show a mean coherence of about 0.5 ; for nominal first year acquisitions with target $\mathrm{HoA}=45 \mathrm{~m}$ (central part), $\bar{\gamma}=0.68$; re-acquisition of affected data takes with HoA $>57$ $\mathrm{m}$ leads to an average coherence of 0.76 , which allows the achievement of the mission requirements.

herence $(\bar{\gamma})$ of about 0.68 is observed, which is expected to provide sufficient phase unwrapping quality, and in very few cases it is below 0.5. Adapting the TanDEM-X acquisition plan, the order of helix satellite formations was reversed, starting with larger baselines, so that data affected by poor performance could be successively reacquired with a smaller baseline, i.e. higher HoA. However, one has to be aware that the height of ambiguity should be kept small in order to meet the height accuracy requirements (according to (3)). The coherence of short baseline acquisitions is depicted on the right part of Figure 4, where an improvement of about $10 \%$ is observed $(\bar{\gamma}=0.76$, and $\gamma$ in no case below 0.6). The combination of additional and nominal acquisitions, by means of multi-baseline phase unwrapping (Lachaise et al., 2007), will then allow to fulfill the final DEM specifications.

\section{Quantization Decorrelation}

In SAR applications, raw data quantization represents an essential aspect, since the number of bits employed for radar signal digitization affects on the 


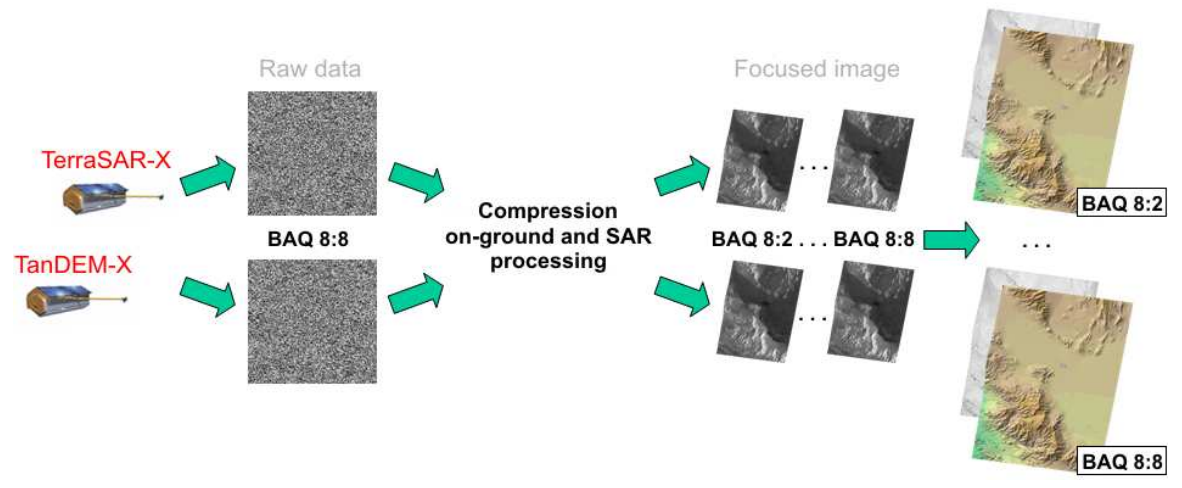

Figure 5: Quantization performance analysis workflow. Raw data commanded with BAQ 8:8 are re-quantized on ground to the available BAQ rates and then processed for the generation of coherence maps and DEMs.

one hand the memory consumption of the satellite and the data volume to be transmitted to the ground, but also on the other hand the performance of the SAR images. On TSX and TDX, the received backscattered signal is first digitized by an 8-bit analog-to-digital converter (ADC) and then further compressed by the block adaptive quantizer. Block adaptive quantization (BAQ) is a lossy data reduction technique which uses local statistics of raw data blocks (with fixed dimension) in order to set the re-quantization decision levels. Possible compression rates are 8:2, 8:3, 8:4, 8:6 and 8:8, where the latter corresponds to BAQ bypass (Mittermayer et al., 2010; Younis et al., 2008). The steps followed to evaluate quantization decorrelation are indicated in Figure 5: raw data commanded with BAQ-bypass have been recompressed on ground by using available $\mathrm{BAQ}$ rates. The obtained products have then been processed into SAR images, allowing the generation of interferograms, coherence maps and final DEMs. Different BAQ settings for the two satellites (e.g. TSX 8:4/TDX 8:3) were investigated, too.

In Figure 6 the interferometric coherence is plotted for all the analyzed scenes and BAQ rates. Test sites have been acquired with different polarization, incidence angles and baselines (the HoA is in the range between 20 $\mathrm{m}$ and $160 \mathrm{~m}$ ). As expected, the fewer bits are employed for quantization, the more coherence degradation is observed for the same test site. For the TanDEM-X mission, BAQ 8:3 (mainly) and 8:4 are planned, which grant at least two global coverages within the nominal mission duration (Krieger et al., 2007) and, at the same time, an acceptable performance degradation. 


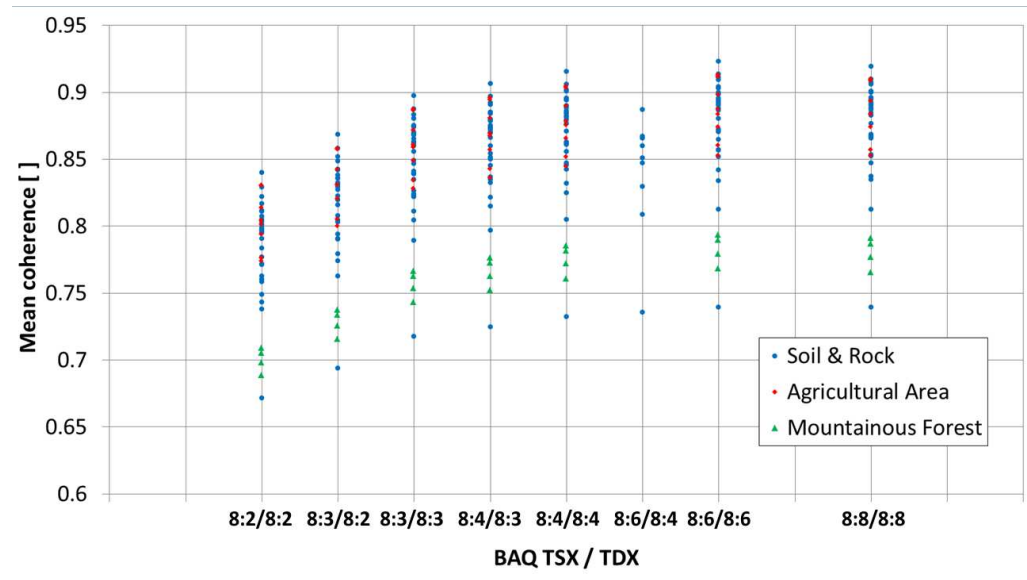

Figure 6: Interferometric coherence for different land cover types and BAQ settings (the compression rate for each channel is given).

Quantization decorrelation relative to the bypass case is given in Figure 7: a coherence loss of about $1 \%$ and $3.5 \%$ is noticed by using 4 and 3 bits/sample, respectively. For typical height of ambiguities (between $40 \mathrm{~m}$ and $50 \mathrm{~m}$ ) and coherence ( 0.6 to 0.9 ), an increase between $5 \mathrm{~cm}$ and $30 \mathrm{~cm}$ in terms of relative height error is expected (according to (3)). The dispersion of the estimated degradations sensitively increases with decreasing quantization rate. Nevertheless, good agreement between theoretic prediction (Krieger et al., 2007) and mean experimental results has been obtained (marked with violet and black lines, respectively). For the global DEM acquisition which will be performed in the second year of the mission, it will be considered to further reduce the quantization rate. As an example, the use of BAQ 8:3 on one satellite and BAQ 8:2 on the other would result in a coherence degradation of about $7 \%$ with reference to the bypass case (and to an additional relative height error between $40 \mathrm{~cm}$ and $70 \mathrm{~cm}$ ), against a data rate reduction of about $17 \%$. Such a solution could be applied on those regions which indicate very good interferometric performance (i.e. $\gamma>0.8$ ). On the other hand, also critical areas showing low coherence (i.e. $\gamma<0.5$ ) could benefit from multiple acquisitions with higher compression, instead of one acquisition with low compression but high data rate (Krieger et al., 2007). 


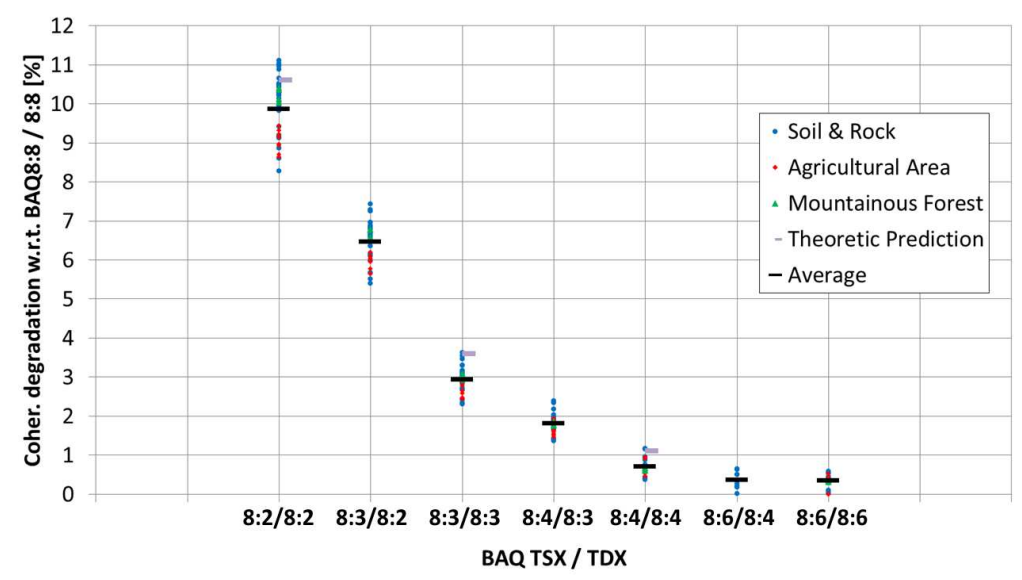

Figure 7: Coherence degradation (in \%) with respect to BAQ 8:8/8:8, for different land cover types. Black and violet lines indicate mean results and theoretic prediction (Krieger et al., 2007), respectively.

\section{Azimuth Ambiguities}

The fourth term in (1) describes the coherence losses contribution due to azimuth and range ambiguities. It can be approximated by (Krieger et al., 2007)

$$
\gamma_{a m b}=\frac{1}{1+\mathrm{RASR}} \cdot \frac{1}{1+\mathrm{AASR}} .
$$

The first term shows the contribution of the range ambiguity to signal ratio (RASR) and the second the contribution of the azimuth ambiguity to signal ratio (AASR). For the TanDEM-X case, decorrelation due to range ambiguities can be neglected (Krieger et al., 2007). The AASR depends strongly on the pulse repetition frequency $(\mathrm{PRF})$ and the azimuth processing bandwidth (PBW). Nominal DEM acquisitions are commanded with PRFs between 3000 $\mathrm{Hz}$ and $4400 \mathrm{~Hz}$. In contrast to the fixed PBW for stripmap mode in the TerraSAR-X mission, the PBW is dynamically adapted to keep the AASR (assuming homogeneous surface) under -20 dB for nominal DEM acquisitions. The PBW adaption can be understood as a $3.11 \mathrm{~dB}$ reduction of AASR for a PRF of $3000 \mathrm{~Hz}$, as depicted in Figure 8. Assuming a non-homogeneous surface with backscatter differences between the target and ambiguous areas, the improvement of coherence is given in Figure 9. Areas with strong backscatter difference of $10 \mathrm{~dB}$ show about $5 \%$ less decorrelation due to az- 

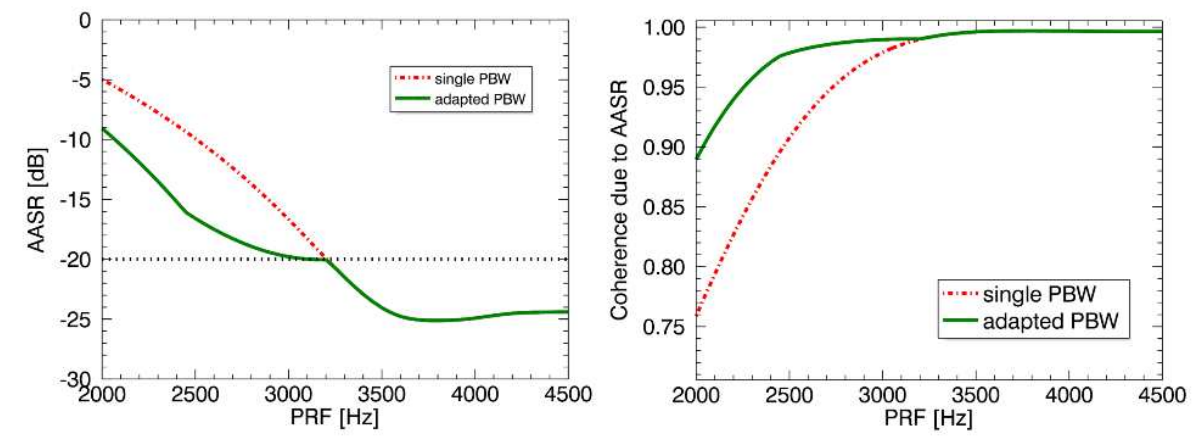

Figure 8: Azimuth ambiguity-to-signal ratio (left) and its impact on the coherence (right).

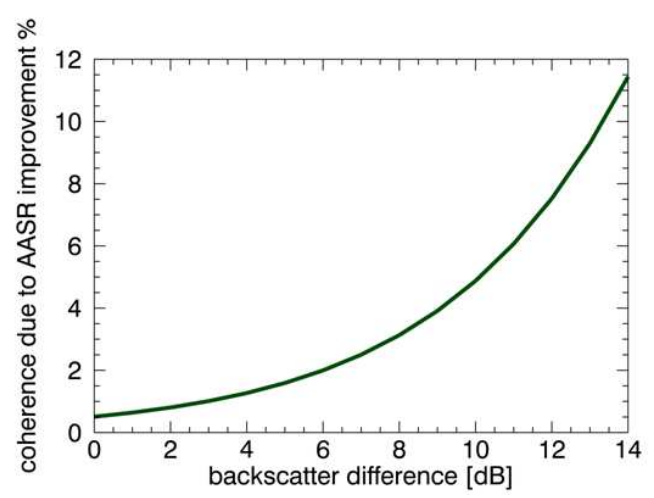

Figure 9: Impact of the PBW adaptation on the coherence for non-homogeneous backscatter distribution $(\mathrm{PRF}=3000 \mathrm{~Hz})$.

imuth ambiguities. For scientific acquisitions such as experimental modes in alternating bistatic or dual-polarization operation, the effective PRF will be even lower (in the order of $2000 \mathrm{~Hz}$ to $3000 \mathrm{~Hz}$ ). The AASR is much smaller with the new PBW approach, so the coherence is higher. For nominal DEM acquisitions azimuth ambiguities cause a coherence loss smaller than $2 \%$.

\section{Polarization Dependence}

The relationship between polarization and interferometric coherence has been deeply investigated during the bistatic Commissioning Phase. Repeated acquisitions in horizontal $(\mathrm{HH})$ and vertical (VV) polarization, with the change of height of ambiguity and incidence angle have been performed over 
Table 2: Test sites for polarization analysis.

\begin{tabular}{|c|c|c|}
\hline Test Site & Land Cover & Latitude Range \\
\hline Salar de Uyuni (Bolivia) & Salt Lake & {$\left[-21^{\circ},-19^{\circ}\right]$} \\
\hline Amazon Forest (Brazil) & Rainforest & {$\left[-12^{\circ},-2^{\circ}\right]$} \\
\hline Borneo Forest (Indonesia) & Tropical Forest & {$\left[-3^{\circ}, 0^{\circ}\right]$} \\
\hline Appalachian Mountains (USA) & Mountainous Deciduous Forest & {$\left[35^{\circ}, 37^{\circ}\right]$} \\
\hline Death Valley (USA) & Soil and Rocks & {$\left[36^{\circ}, 37^{\circ}\right]$} \\
\hline Black Rock Desert (USA) & Soil and Rocks & {$\left[40^{\circ}, 42^{\circ}\right]$} \\
\hline Iowa (USA) & Agricultural Area & {$\left[41^{\circ}, 43^{\circ}\right]$} \\
\hline Oregon (USA) & Mountainous Evergreen Forest & {$\left[42^{\circ}, 46^{\circ}\right]$} \\
\hline Alberta, Ontario, Saskatchewan (Canada) & Boreal Forest & {$\left[48^{\circ}, 59^{\circ}\right]$} \\
\hline Krycklan (Sweden) & Boreal Forest & {$\left[64^{\circ}, 65^{\circ}\right]$} \\
\hline
\end{tabular}

areas showing different land cover types. The used test sites are listed in Table 2. In total, about three hundred scenes were investigated. Figure 10 shows the coherence over argument of latitude, for $\mathrm{HH}$ and VV channel: on average, almost no difference can be noticed $\left(\bar{\gamma}_{\mathrm{HH}}=0.729\right.$ and $\left.\bar{\gamma}_{\mathrm{VV}}=0.723\right)$. Better coherence is observed at higher latitudes as a result from the different vegetation characteristics and from the varying HoAs due to the fixed Helix formation (Krieger et al., 2007), according to which the effective baseline is bigger around the equator and becomes smaller at higher latitudes. Since the mean backscatter observed in the data is slightly higher in $\mathrm{HH}$, the horizontal polarization has been selected for the global DEM acquisition.

\section{Long-Term Performance Monitoring}

Since the beginning of 2011, dedicated acquisitions for long-term monitoring of the interferometric performance of the TanDEM-X mission have been carried out. Test sites with different land and topography characteristics are regularly acquired, in order to investigate possible changes of coherence due to formation geometry (baseline slowly decreasing during the time) and/or scene target modification. On the other hand, for each test site all radar parameters have been kept identical. In Figure 11 the coherence over height of ambiguity (HoA) is depicted for areas characterized by dense rainforest, flat and hilly tropical forest, and soil and rock cover. It can be clearly seen that the specific acquisition geometry plays a key-role in interferometric performance for regions showing strong volume decorrelation phenomena (as 


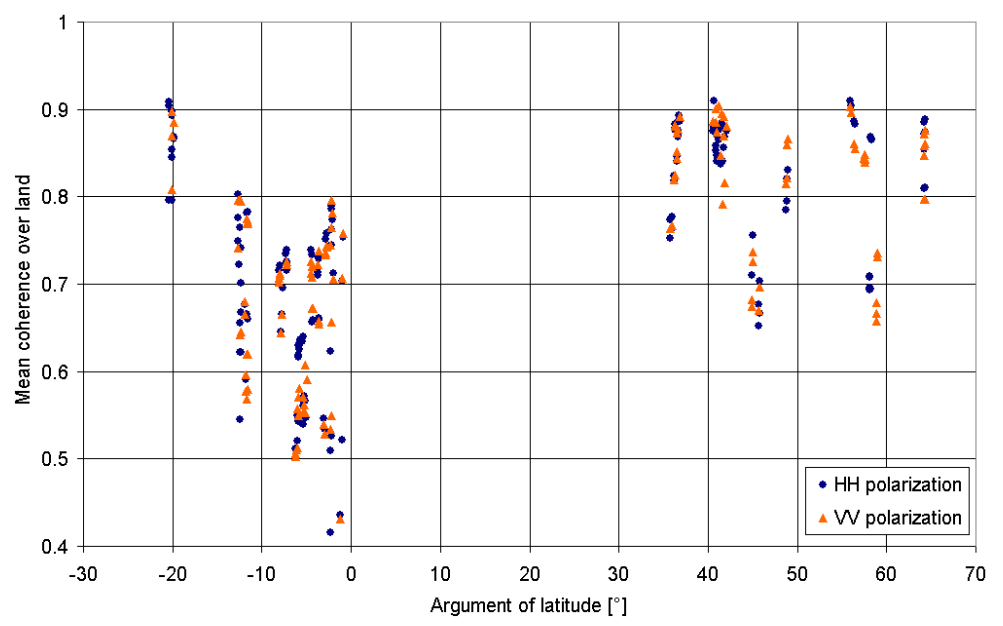

Figure 10: Average coherence for $\mathrm{HH}$ and VV polarization over argument of latitude: both polarizations yield similar interferometric performance (on average, $\gamma_{\mathrm{HH}}=0.729$ and $\left.\gamma_{\mathrm{VV}}=0.723\right)$. Worse coherence for latitude between $-15^{\circ}$ and $0^{\circ}$ are obtained as a results from stronger volume decorrelation effects over tropical and rainforest, and from the varying HoAs due to the fixed Helix formation (Krieger et al., 2007).

observed in Section 3). This is evident in the case of the "Amazon Rainforest 1" site (dark blue circles and squares), acquired with different incidence angles, thus covering a wide range of HoA. A coherence improvement ( $15 \%$ to $20 \%$ ) is also noticed for the "Indonesia mountainous" and "Amazon Rainforest 2" sites (light blue and green circles, respectively), mainly in the height of ambiguity range between $30 \mathrm{~m}$ and $75 \mathrm{~m}$. For all other test sites (flat tropical forest, soil and rock) and for smaller baselines, a remarkable stability over time (and HoA) can be observed. Such analyses will be continued during the TanDEM-X DEM acquisition phase, providing then a complete and consistent basis for performance studies of future missions.

\section{Global DEM Acquisition Status}

The TanDEM-X operational DEM acquisition has started on December 12, 2010. From that day on, more than 150,000 bistatic scenes have been processed and performance parameters have been assessed (Fritz et al., 2011). A view of the distribution of the interferometric coherence over the globe for acquisitions processed until April 2012 is given in Figure 12: according to the TanDEM-X acquisition plan a nearly complete coverage of all landmasses has 


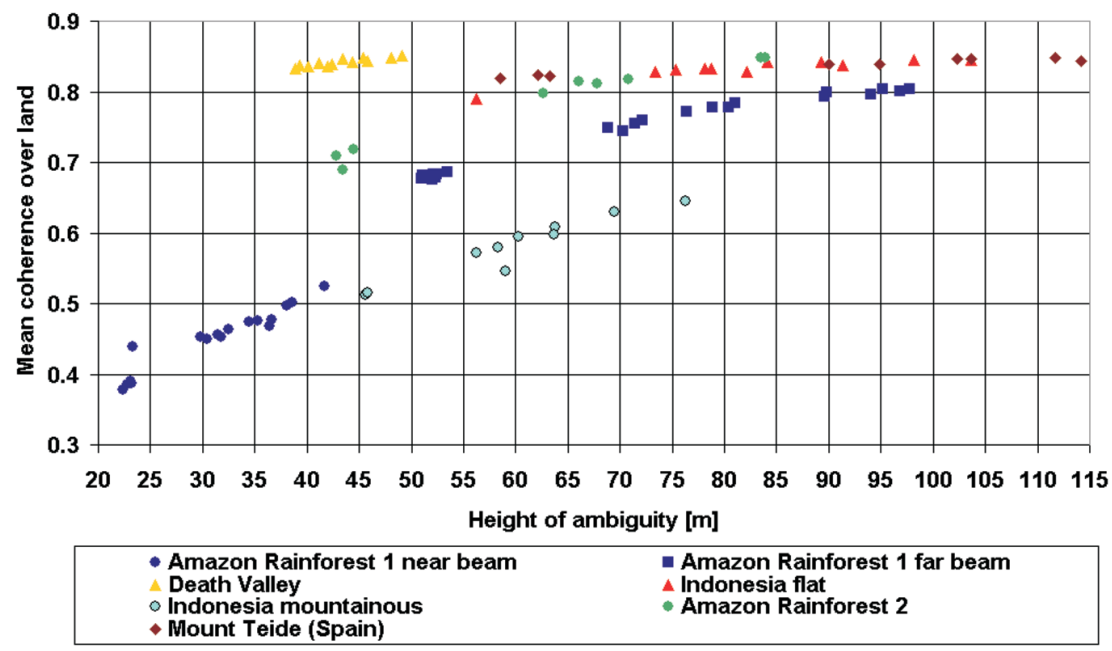

Figure 11: Coherence over height of ambiguity for long term performance monitoring acquisitions. Considerable performance improvement is observed in the case of densely forested and mountainous areas, for $\mathrm{HoA} \in[30,75] \mathrm{m}$.

been achieved for latitudes greater than $-60^{\circ}$, while the Antarctic region will be entirely acquired in a later mission phase. In Figure 13 the cumulative density of coherence is depicted, and the obtained mean coherence over all scenes is of about 0.75 . In particular, more than $92 \%$ of the processed DEM acquisitions are characterized by a mean land coherence higher than 0.6 , and $46 \%$ even higher than 0.8 . For many of these areas, a single acquisition would be already sufficient to achieve the specified relative height accuracy. Very good performance is shown also by phase unwrapping quality indicators: mean percentage of single cuts and phase residues (Bamler and Hartl, 1998) are around $0.4 \%$ and $0.5 \%$, respectively, which is expected to provide more than sufficient DEM quality (Suchandt and Eineder, 2003).

\section{Conclusions and Future Activities}

This paper presented a thorough coherence evaluation of TanDEM-X interferometric data acquired during the bistatic Commissioning Phase and within the first year of operational acquisition. It was proved that sites characterized by different backscatter intensities show satisfactory and reproducible interferometric performance, and a strategy to handle with those areas strongly affected by SNR decorrelation has been presented. Due to 


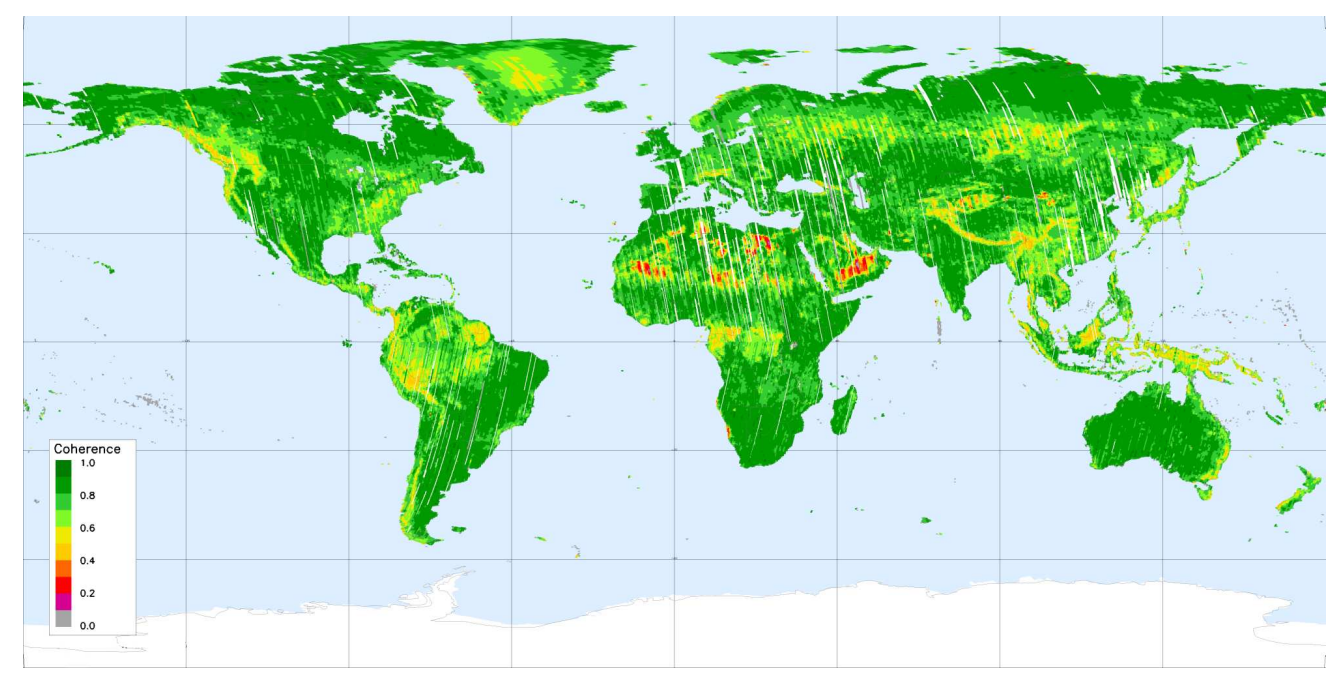

Figure 12: Interferometric coherence and coverage distribution of the already processed global DEM acquisitions, acquired from December 2010 to end of March 2012.

volume decorrelation effects, dense and hilly forests require additional acquisitions with an optimized imaging geometry (i.e. smaller baselines) and, thus, the DEM acquisition plan has been consequently adapted, by increasing the target HoA to $45 \mathrm{~m}$ and planning additional acquisitions with a height of ambiguity over $60 \mathrm{~m}$ in critical areas. Quantization causes acceptable performance loss and good agreement has been observed between experimental results and theoretical prediction (Krieger et al., 2007). The opportunity to further increase the raw data compression rate (e.g. different rates between the two satellites) in order to improve the overall DEM performance will be further investigated. It was shown that for $\mathrm{HH}$ and VV polarizations almost the same interferometric performance is obtained. Since the mean backscatter is slightly higher in horizontal polarization, this was selected for the global DEM acquisition. Long-term performance monitoring by means of repeat-pass acquisitions on defined sites confirmed that the specific acquisition geometry has a significant impact on the interferometric coherence for densely forested areas. For other land cover types or HoA $>75 \mathrm{~m}$, stable performance is observed. Looking at the statistics of the first global DEM acquisition, promising results in terms of coherence and phase unwrapping quality indicators are observed so far, showing that the TanDEM-X mission is able to provide the remote sensing scientific community with a unique data set to exploit the capability of the first bistatic SAR mission and to apply 


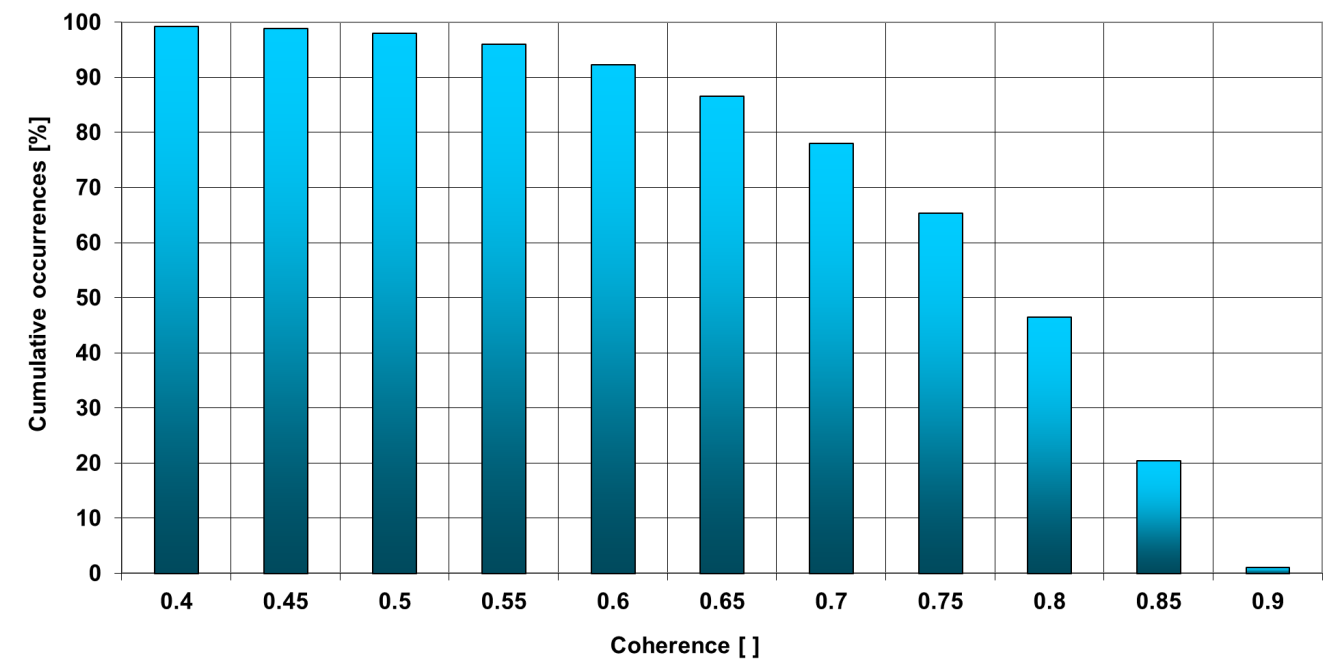

Figure 13: Cumulative coherence distribution of TanDEM-X operational DEM acquisitions: each bar depicts the percentage of scenes showing coherence higher than the indicated value.

these innovative techniques for a broad range of commercial and scientific applications.

\section{Acknowledgments}

The authors would like to thank the anonymous reviewers for their valuable comments and suggestions that helped to improve this paper.

The TanDEM-X project is partly funded by the German Federal Ministry for Economics and Technology (Förderkennzeichen $50 \mathrm{EE} \mathrm{1035).}$

\section{References}

Bamler, R., Hartl, P., 1998. Synthetic aperture radar interferometry. Inverse Problems 14, 1-54.

Breit, H., Younis, M., Balss, U., Niedermeier, A., Grigorov, C., Hueso Gonzalez, J., Krieger, G., Eineder, M., Fritz, T., 2011. Bistatic synchronization and processing of TanDEM-X data. Proc. IGARSS, Vancouver, Canada, 24-29 July, pp. 2424-2427. 
DEM products specification, 2012. EOC - TanDEM-X Ground Segment - DEM Products Specification Document, http://tandemx-science.dlr. de/pdfs/TD-GS-PS-0021_DEM-Product-Specification_v1.7.pdf . (Accessed 1 February, 2012)

Fritz, T., Rossi, C., Yague-Martinez, N., Rodriguez-Gonzalez, F., Lachaise, M., Breit, H., 2011. Interferometric Processing of TanDEM-X data. Proc. IGARSS, Vancouver, Canada, 24-29 July, pp. 2428-2431.

Hueso Gonzalez, J., Krieger, G., Walter Anthony, J., Bachmann, M., Schrank, D., Schwerdt, M., De Zan, F., Rodriguez-Cassola, M., 2011. Baseline and bistatic system calibration in the TanDEM-X mission. Proc. IGARSS, Vancouver, Canada, 24-29 July, 4 p. (on CD-ROM).

Just, D., Bamler, R., 1994. Phase statistics of interferograms with applications to synthetic aperture radar. Applied Optics 33 (20), 4361-4368.

Krieger, G., Moreira, A., Fiedler, H., Hajnsek, I., Werner, M., Younis, M., Zink, M., 2007. TanDEM-X: a satellite formation for high-resolution SAR interferometry. IEEE Transactions on Geoscience and Remote Sensing 45 (11), 3317-3341.

Lachaise, M., Eineder, M., Fritz, T., 2007. Multi baseline SAR acquisition concepts and phase unwrapping algorithms for the TanDEM-X mission. Proc. IGARSS, Barcelona, Spain, 23-27 July, pp. 5272-5276.

Lee, J.-S., Hoppel, K. W., Mango, S. A., Miller, A. R., 1994. Intensity and phase statistics of multilook polarimetric and interferometric SAR imagery. IEEE Transactions on Geoscience and Remote Sensing 32 (5), 1017-1028.

Mittermayer, J., Younis, M., Metzig, R., Wollstadt, S., Martinez, J. M., Meta, A., 2010. TerraSAR-X system performance characterization and verification. IEEE Transactions on Geoscience and Remote Sensing 48 (2), 660-676.

Moreira, A., Krieger, G., Hajnsek, I., Werner, M., Hounam, D., Riegger, S., Settelmeyer, E., 2004. TanDEM-X: a TerraSAR-X add-on satellite for single-pass SAR interferometry. Proc. IGARSS, Anchorage, Alaska, 20-24 
September, pp. 1000-1003.

Rodriguez, E., Morris, C. H., Belz, J. E., 2006. A global assessment of the SRTM performance. Photogrammetric Engineering and Remote Sensing 72 (3), 249-260.

Suchandt, S., Eineder, M., 2003. Experience with SRTM/X-SAR phase unwrapping using the minimum cost flow method. Proc. IGARSS, Toulouse, France, 21-25 July, pp. 4380-4382.

Treuhaft, R. N., Siqueira, P. R., 2000. The vertical structure of vegetated land surfaces from interferometric and polarimetric radar. Radio Science 35 (1), 131-177.

Werner, M., 2001. Shuttle Radar Topography Mission (SRTM): mission overview. Journal of Telecommunication (Frequenz) 55 (3-4), 75-79.

Younis, M., Böer, J., Ortega-Míguez, C., Schulze, D., Huber, S., Mittermayer, J., 2008. Determining the optimum compromise between SAR data compression and radiometric performance - An approach based on the analysis of TerraSAR-X data -. Proc. IGARSS, Boston, Massachusetts, 6-11 July, pp. 107-110.

Zebker, H. A., Farr, T. G., Salazar, R. P., Dixon, T. H., 1994. Mapping the world's topography using radar interferometry: the TOPSAT mission. IEEE Transactions on Geoscience and Remote Sensing 82 (12), 1774-1786.

Zebker, H. A., Villasenor, J., 1992. Decorrelation in interferometric radar echoes. IEEE Transactions on Geoscience and Remote Sensing 30 (5), 950959. 